\title{
Two percolation thresholds and remarkably high dielectric permittivity in pristine carbon nanotube/elastomer composites
}

\author{
Khurram Shehzad • Ayaz Ali Hakro • You Zeng • \\ Shang-Hong Yao • Yi Xiao-Hong • Muhammad Mumtaz • \\ Kashif Nadeem $\cdot$ Nasir Said Khisro $\cdot$ Zhi-Min Dang
}

Received: 25 November 2014/ Accepted: 19 January 2015/Published online: 31 January 2015

(C) The Author(s) 2015. This article is published with open access at Springerlink.com

\begin{abstract}
Pristine carbon nanotube (CNT)/elastomer composites were fabricated using pristine multi-walled carbon nanotubes and a thermoplastic elastomer. These composites exhibited a unique phenomenon of two electrical percolation thresholds that invoked very high dielectric values for the resulting composites. The first percolation was associated with a relatively low dielectric constant value of about 100 , while in the vicinity of the second percolation threshold a very high dielectric constant value of 8,000 was achieved. The presence of two percolation thresholds was attributed to the unique distribution patterns of CNTs that ensued in a CNT/elastomer composite system with unique electrical properties.
\end{abstract}

Keywords Carbon nanotubes - Thermoplastic elastomer . Two percolation thresholds - Dielectric permittivity . Electrical conductivity

K. Shehzad $(\bowtie)$ · A. A. Hakro · N. S. Khisro

Department of Information Science and Electronic

Engineering, Zhejiang University, Hangzhou 310027,

People's Republic of China

e-mail: khurrams@mail.zju.edu.cn

K. Shehzad · S.-H. Yao

State Key Laboratory of Chemical Resource

Engineering, Beijing University of Chemical Technology,

Beijing 100029, China

\section{Y. Zeng}

Institute of Metal Research, Chinese Academy of Science,

Shenyang, China

S.-H. Yao

Grenoble Electrical Engineering Laboratory (G2ELab), Joseph Fourier University (UJF), CNRS, 25 Rue des Martyrs,

38042 Grenoble, France

\section{Introduction}

Dielectric elastomers (DEs), due to their large-strain actuation under the application of a high electric field or to convert mechanical energy into electricity, have found a wide range of applications, such as refreshable braille displays (Niu et al. 2012; Yu et al. 2009), tunable lenses (Carpi et al. 2011), cell stretchers (Akbari and Shea 2012), soft robots (Pei et al. 2003) and buoy generators (Chiba et al. 2008). A high dielectric constant is desirable for the mentioned applications of DEs, but the existing DE materials (Keplinger et al. 2012; Kofod et al. 2003; Stoyanov et al. 2013) have rather low dielectric constants. To substantially improve the electrical properties, conducting fillers such as metals, conducting polymers, carbon nanotubes (CNTs) and carbon black are usually employed (Dang et al. 2011; Hu et al. 2014; Lu et al. 2006; Shehzad et al. 2013b, c, 2014; Wang et al. 2013). Among these

\section{Y. Xiao-Hong}

China Center for Industrial Security Research, Beijing Jiao tong

University, Beijing, China

M. Mumtaz $\cdot$ K. Nadeem

Beijing National Laboratory for Condensed Matter Physics, Institute of Physics, Chinese Academy of Science, Beijing, Haidian 100084, China

\section{Mumtaz $\cdot$ K. Nadeem}

Materials Research Laboratory, Department of Physics

FBAS, International Islamic University (IIU) Islamabad, Islamabad 44000, Pakistan

\section{Z.-M. Dang ( $\square)$}

Department of Polymer Science and Engineering, School of Chemistry and Biological Engineering, University of Science and Technology Beijing, Beijing 100083, China

e-mail: dangzm@ustb.edu.cn 
fillers, CNTs stand out because of their high surface area and exotic electrical, mechanical and thermal properties. Another major advantage of CNTs is that due to their high aspect ratio, they achieve the percolation threshold at relatively lower filler concentrations (Sandler et al. 2003). However, the potential of CNTs to serve as conductive filler is partially eclipsed due to the strong inter-filler force that leads to aggregation of CNTs and the poor interface between CNT and polymer. To better disperse the CNTs and subsequently obtain superior electrical properties of CNT/polymer composites, researchers like Dang et al. (2007b) and Liu et al. (2011) have come up with chemical approaches to modify the surface of CNTs. However, the chemical modification approach of CNTs have inherent disadvantage of involving complex and potentially hazardous chemical routes. Moreover, these chemical modifications can partially destroy the structure of CNTs resulting in the impediment of their electronic properties. Furthermore, chemical modification does not always guarantee improved dielectric properties as in the case of Liu et al. (2011) where, despite the chemical modification of CNTs, the dielectric permittivity of the composites was below 100 even at the CNT concentration of 20 vol. $\%$.

Keeping in view the disadvantages of chemical modification of CNTs, there is a strong need to better exploit the electrical properties of CNTs as filler by using them in their pristine form. Using the CNTs in the pristine form will not only preserve their structural integrity, but also simplify the composite fabrication process. Here, we report a pristine CNT/elastomer composite where CNTs without any surface modifications were used. Furthermore, we use a polypropylene-based thermoplastic elastomer (PP/EPDM), which allowed multiple melt processing. So, the process was fast, simple and easily scalable. CNT topologies that arise from melt mixing of pristine CNTs lead to remarkably high dielectric permittivity values $(\sim 8,000)$. Two distinct structural transitions of CNT networks were observed that were deemed responsible for such extraordinarily high dielectric permittivity values.

\section{Experimental}

For composite fabrication, multi-walled carbon nanotubes (MWCNTs), prepared by chemical-vapor deposition method with a diameter range of $10-30 \mathrm{~nm}$, length range of 5-15 um and average aspect ratio of 833 (Fig. 4a), were supplied by Shenzhen Nanotech Port Company, China. Composites of the polymer and MWCNT were fabricated and tested by the already described procedures (Shehzad et al. 2013a). The only difference was that in reference 18 a flash mold was used for compression molding to make thin composite sheets, while in this case a landed positive-type mold was employed. Five sets of each composition were tested to minimize the experimental error, and average values of dielectric permittivity and electrical conductivity were obtained. AC conductivity and dielectric permittivity of the samples were measured using an Agilent HP 4194A impedance analyzer. Images of fractured cross-surfaces of different composites were obtained on an environmental scanning electron microscope of type Philips-FEI XL-30.

\section{Results and discussion}

Figure 1a shows the effect of MWCNT (hereafter referred to as CNT) concentration on the dielectric permittivity values of the elastomer composites. On the larger dielectric scale ( $y$-axis) of maximum value of up to the 9,000 , a transition at 0.13 volume fraction of CNT is apparent suggesting the formation of percolation networks. Near the critical concentration of CNTs ( 0.13 volume fraction), the dielectric permittivity of the CNT/elastomer composites reached as high as 8,000 , which amounts to an increase of about 2,400-fold over the dielectric permittivity of pure elastomer (3.31). This sudden and extraordinary rise in dielectric permittivity values of the composites is the result of interfacial polarization at the interface between elastomer and CNTs caused by the presence of a large number of micro-capacitor structures formed between two neighboring CNTs separated by an insulating matrix. The critical value of 0.13 was not an observational value, but in fact was obtained using the best fit of the power law given in Eq. 1.

$\varepsilon=\varepsilon_{\mathrm{o}}[((f \mathrm{c}-f) / f \mathrm{c})]^{-q}$,

where $\varepsilon_{\mathrm{O}}$ is the dielectric permittivity of the insulator matrix, $f$ is the volume fraction of the conductive particles, $f \mathrm{c}$ is the percolation threshold or the critical concentration and $\mathrm{q}$ is a critical exponent. With the best linear fit of Eq. 1, the value of $f \mathrm{c}$ was 0.13 and its corresponding $\mathrm{q}$ value was about 2.8, which is higher than the universal $q$ value of $0.8-1$, suggesting a deviation from the universal behavior (Ce-Wen 1993). When the dielectric axis was scaled down (up to the value of 300), a second transition became apparent near the CNT volume fraction of 0.07 (See Fig. 1b). The transition at 0.07 produced dielectric values of around 100. The existence of two transitions or percolation thresholds at 0.07 and 0.13 was puzzling, as there has been no previous experimental report of two percolation thresholds in CNT/polymer composite systems. The theoretical plot of Eq. 1 (see black dotted line in Fig. 1d), considering the percolation threshold at 0.07 , also suggests a decrease in the dielectric permittivity of the composites with increase in filler concentration in the post-percolation threshold region. This theoretical outcome of 
Fig. 1 a, b, d Dielectric permittivity; c electrical conductivity of CNT/elastomer composites as a function of CNT loadings
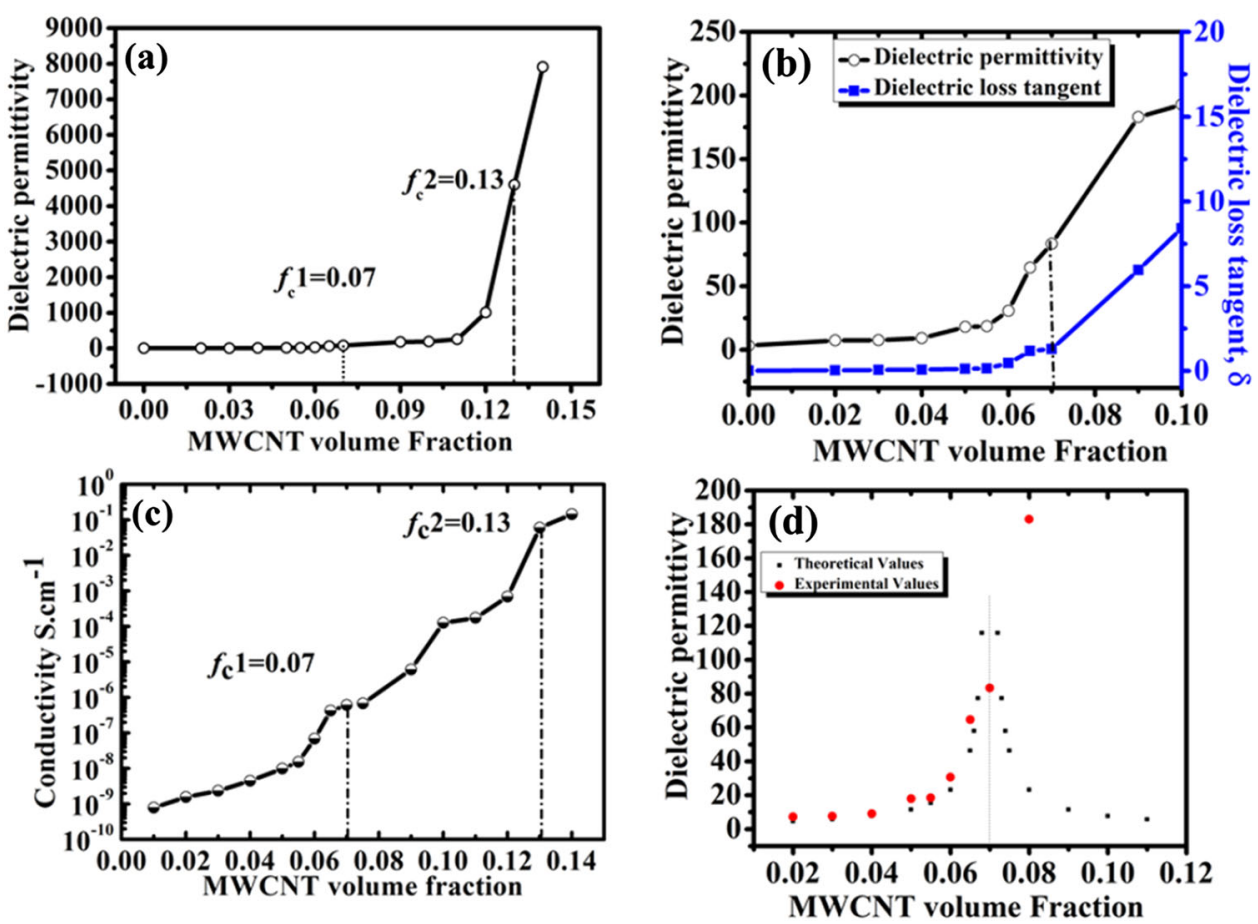

post-percolation threshold trend of monotonic decrease in dielectric values of practical CNT/polymer composites has been reported in previous reports (Dang et al. 2007a; Wang and Dang 2005). The mechanism of this decrease is that in the post-percolation region, composites become conductors and resultantly have a large leakage current that destroys the dielectric properties of the composites. However, contrary to theoretical predictions, in our case the dielectric permittivity kept increasing even after the first percolation threshold (see red dotted line in Figs. 1d, 2) and went on to experience another percolation at 0.13 . To confirm the existence of two percolations in our system, the fitting constants q given in Eq. 1 can be used as a testing tool. For the 3D percolating composites, the universal value of $q$ is in the range of $0.8-1(\mathrm{Ce}-\mathrm{Wen} 1993)$. The value of $q$ calculated, while ignoring the minor transition at 0.07 and considering only the single transition at 0.13 , was 2.18 , far from the universal value range of $0.8-1$. However, when we divided the curve into two percolation steps at 0.07 and 0.13 and calculated the value of $q$ for CNT concentrations from 0 to 0.07 , the value of $q$ attained (1.04) was approximate of the universal value range of $0.8-1$. For the second transition, we took 0.07 as the starting point and calculated the $\mathrm{q}$ value in the range of $0.07-0.13$ volume fraction of CNTs. Again, the q value (1.24) for this transition was closer to the universal value range of $0.8-1$. Convergence of the $q$ value toward the universal values when two percolation thresholds instead of a single percolation threshold were considered is suggestive of the presence of two percolation thresholds. No previous study, to the best of our knowledge, has reported the two percolation thresholds in CNT/polymer composites. However,
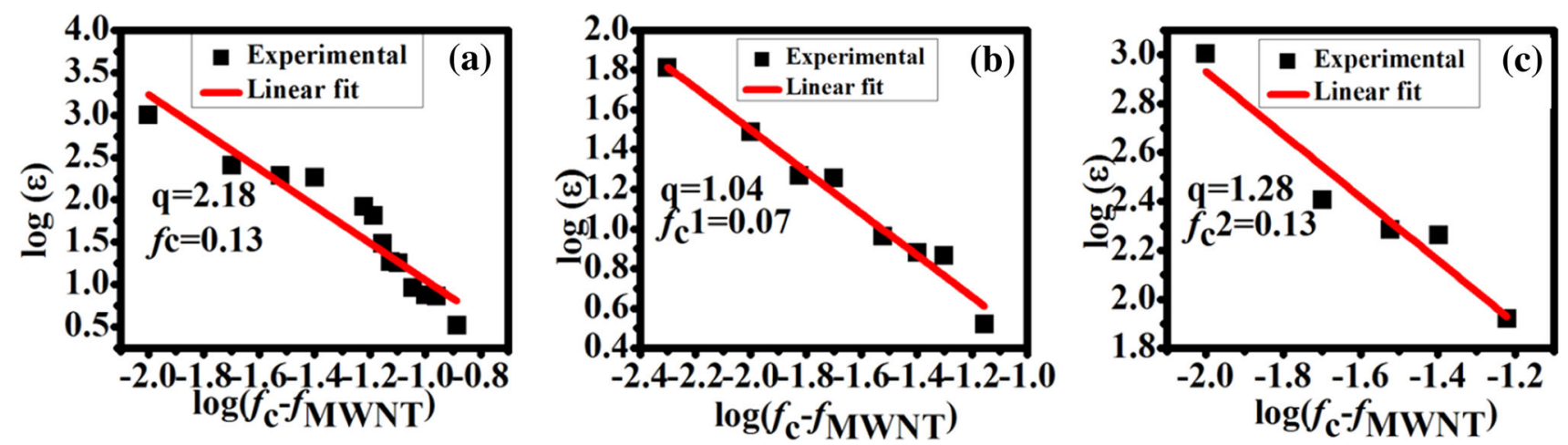

Fig. 2 Linear fittings of Eq. 1: a considering only one percolation threshold at 0.13 volume fraction of MWCNTs; b, c considering two percolation thresholds at 0.07 and 0.013 volume fractions of MWCNTs, respectively 


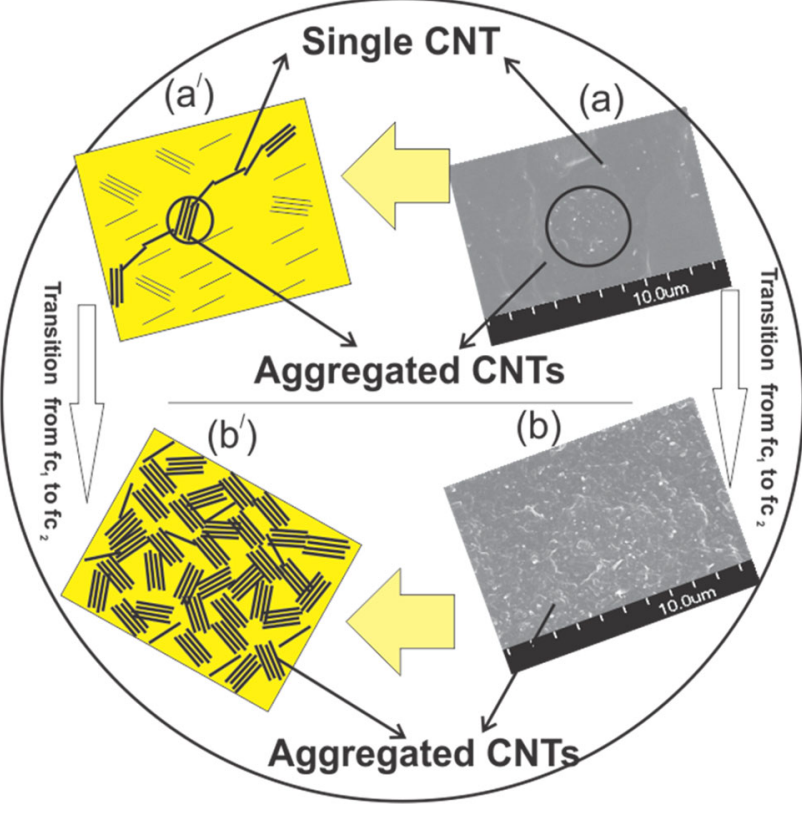

Fig. 3 a, b SEM images of CNT/elastomer composites at 0.07 and 0.013 volume fraction CNT loadings. a', b' Schematics of CNT networks at 0.07 and 0.013 volume fraction CNT loadings

the presence of two percolation thresholds has been reported for the $\mathrm{SiC} /$ rubber composite system by Nettelblad et al. (2003), where the electrical conductivity curve displayed not one, but two percolation thresholds. The authors have explained this phenomenon, experimentally and by simulations, by taking into account two different types of contacts (represented by edge and face connections) between conducting $\mathrm{SiC}$ particles.

In our case, two percolation thresholds were the result of different types of CNT networks in the elastomer matrix at each percolation threshold. Two different types of networks at each percolation threshold probably originated from the unique distribution of CNTs. We believe that the first percolation threshold is the result of percolating networks that are the result of zones of high and low density of CNTs per unit volume in the elastomer matrix. It is clear from the SEM images and schematics in Fig. 3a and a', respectively, that at first percolation thresholds, there are zones of low density of CNTs per unit volume, where isolated CNTs can be viewed, as well as zones of high CNT density per unit volume, where CNTs exist as "aggregates". Clearer high-magnification SEM images of zones with high- (aggregated CNTs) and low-density CNTs (individual CNTs) in CNT/elastomer composites at 0.07 volume fraction CNT loadings are given in Fig. 4b and c, respectively. It is important to mention here that the word "aggregate" does not mean that CNTs are in direct contact with each other. In fact, on nanoscale, even in these aggregate structures, CNTs are separated by a very thin polymer films and thus can be considered as dispersed. At the second percolation thresholds, CNT networks were made only of CNT "aggregates" (high-density CNT zones) (Fig. 3b, $b^{\prime}$ ) as at this stage there were no prominent lowdensity CNT zones. Percolating networks made of aggregated structure at the second percolation threshold resulted in a very high dielectric permittivity value $(\sim 8,000)$ of the composites. This dielectric permittivity of our pristine CNT/elastomer composites was much higher compared to already reported chemically functionalized CNT-based composites where the maximum dielectric permittivity value obtained was no more than 1,000 (Galantini et al. 2013; Liu et al. 2011; Yadav et al. 2013; Yang et al. 2009)

The unique structural transition in our composites also resulted in a unique percolation curve for electrical conductivity (Fig. 1c). The electrical conductivity curve was wide and quasi-linear compared with classical theoretical curves and other reported curves that are usually sharp in nature (Sandler et al. 2003; Zeng et al. 2010). This quasilinear curve not only provides a wide window of conductivity to work with, a luxury not associated with sharp percolation curves, but was also indirectly responsible for high dielectric permittivity values and the second percolation threshold. If the conductivity curve was sharp and it would have achieved the plateau conductivity soon after the first percolation threshold $\left(f_{\mathrm{c} 1} 1\right)$, then composites would
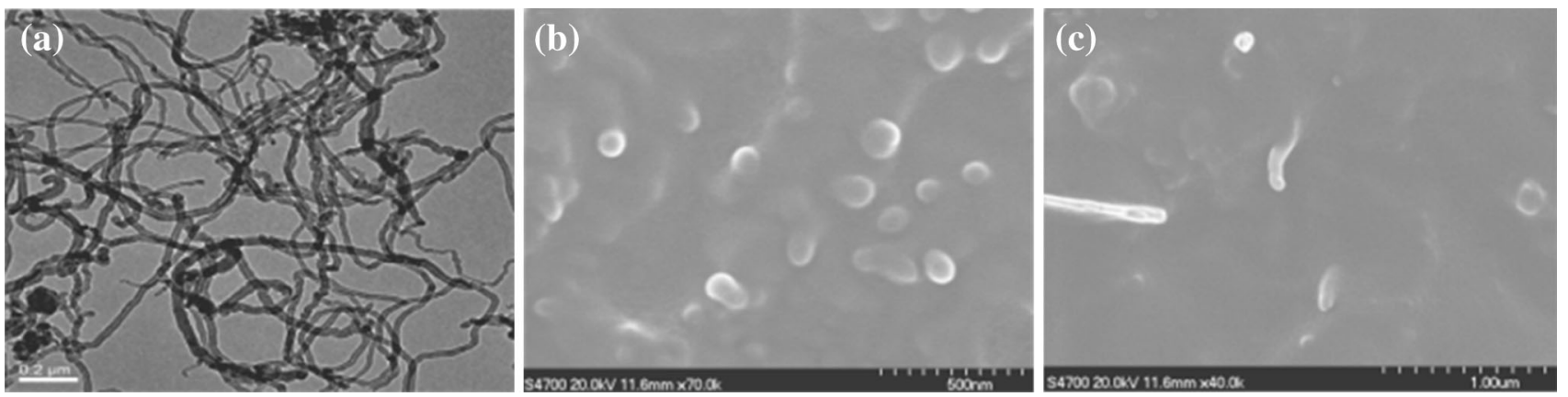

Fig. 4 a TEM images of CNTs using: b higher-magnification cross-sectional SEM image of "aggregated" CNTs and c higher-magnification cross-sectional SEM image of "isolated" CNTs in CNT/elastomer composites at 0.07 volume fraction 
have become conductive and there was no possibility of further increase in the dielectric permittivity values. However, in our case, post-first percolation conductivity increased gradually from $10^{-6} \mathrm{~S} / \mathrm{cm}$ at 0.07 volume fraction of CNT $(f \mathrm{c} 1)$ to $10^{-1} \mathrm{~S} / \mathrm{cm}$ at 0.13 volume fraction of CNT $(f \mathrm{c} 2)$, allowing the possibility of further increase in the dielectric permittivity of the composites that subsequently resulted in another transition at 0.13 volume friction.

\section{Conclusions}

In conclusion, the novel pristine CNT/elastomer composites system presented in this study is characterized by two percolation thresholds with high dielectric permittivity value and quasi-linear electrical conductivity curve. The analysis of the composites suggests that the two percolation thresholds that resulted in unique electrical properties were the result of the unique distribution patterns of CNTs in the elastomer matrix. The first percolation threshold was the result of the networks made of low- and high-density CNT regions, while the second percolation was attributed to the networks of high-density CNT aggregates.

Acknowledgments This work was financially supported by NSF of China (grant Nos. 51377010 and 51250110536), State Ministry of Education of China through Doctor Project (Grant No. 20130006130002) and the National Basic Research Program of China (973 Program) (Grant No. 2014CB239503).

Open Access This article is distributed under the terms of the Creative Commons Attribution License which permits any use, distribution, and reproduction in any medium, provided the original author(s) and the source are credited.

\section{References}

Akbari S, Shea HR (2012) Microfabrication and characterization of an array of dielectric elastomer actuators generating uniaxial strain to stretch individual cells. J Micromech Microeng 22:045020

Carpi F, Frediani G, Turco S, De Rossi D (2011) Bioinspired Tunable Lens with Muscle-Like Electroactive Elastomers. Adv Funct Mater 21:4152-4158. doi:10.1002/adfm.201101253

Ce-Wen N (1993) Physics of inhomogeneous inorganic materials. Prog Mater Sci 37:1-116

Chiba S, Waki M, Kornbluh R, Pelrine R (2008) Innovative power generators for energy harvesting using electroactive polymer artificial muscles. pp 692715-692719

Dang Z-M, Wu J-P, Xu H-P, Yao S-H, Jiang M-J, Bai J (2007a) Dielectric properties of upright carbon fiber filled poly (vinylidene fluoride) composite with low percolation threshold and weak temperature dependence. Appl Phys Lett 91:072912072912-072912-072913

Dang ZM, Wang L, Yin Y, Zhang Q, Lei QQ (2007b) Giant Dielectric Permittivities in Functionalized Carbon-Nanotube/
Electroactive-Polymer Nanocomposites. Adv Mater s 19:852-857. doi:10.1002/adma.200600703

Dang ZM, Shehzad K, Zha JW, Nie J, Shi CY (2011) Complementary percolation characteristics of carbon fillers based electrically percolative thermoplastic elastomer composites. Compos Sci Technol 72(1):28-35

Galantini F, Bianchi S, Castelvetro V, Gallone G (2013) Functionalized carbon nanotubes as a filler for dielectric elastomer composites with improved actuation performance. Smart Mater Struct 22:055025

Hu W, Zhang SN, Niu X, Liu C, Pei Q (2014) An aluminum nanoparticle-acrylate copolymer nanocomposite as a dielectric elastomer with a high dielectric constant. J Mat Chem C 2:1658-1666. doi:10.1039/C3TC31929F

Keplinger C, Li T, Baumgartner R, Suo Z, Bauer S (2012) Harnessing snap-through instability in soft dielectrics to achieve giant voltage-triggered deformation. Soft Matter 8:285-288. doi:10. 1039/C1SM06736B

Kofod G, Sommer-Larsen P, Kornbluh R, Pelrine R (2003) Actuation Response of Polyacrylate Dielectric Elastomers. J Intell Mater Syst Struct 14:787-793. doi:10.1177/104538903039260

Liu H, Shen Y, Song Y, Nan C-W, Lin Y, Yang X (2011) Carbon Nanotube Array/Polymer Core/Shell Structured Composites with High Dielectric Permittivity, Low Dielectric Loss, and Large Energy Density. Adv Mater (Weinheim, Ger) 23:5104-5108. doi:10.1002/adma.201102079

Lu J, Moon K-S, Xu J, Wong C (2006) Synthesis and dielectric properties of novel high-K polymer composites containing in situ formed silver nanoparticles for embedded capacitor applications. J Mater Chem 16:1543-1548

Nettelblad B, Mårtensson E, Önneby C, Gäfvert U, Gustafsson A (2003) Two percolation thresholds due to geometrical effects: experimental and simulated results. J Phys D Appl Phys 36:399

Niu X, Yang X, Brochu P, Stoyanov H, Yun S, Yu Z, Pei Q (2012) Bistable Large-Strain Actuation of Interpenetrating Polymer Networks. Adv Mater 24:6513-6519. doi:10.1002/adma.201202876

Pei Q, Rosenthal MA, Pelrine R, Stanford S, Kornbluh RD (2003) Multifunctional electroelastomer roll actuators and their application for biomimetic walking robots, pp 281-290

Sandler J, Kirk J, Kinloch I, Shaffer M, Windle A (2003) Ultra-low electrical percolation threshold in carbon-nanotube-epoxy composites. Polymer 44:5893-5899

Shehzad K, Dang Z-M, Ahmad MN, Sagar RUR, Butt S, Farooq MU, Wang T-B (2013a) Effects of carbon nanotubes aspect ratio on the qualitative and quantitative aspects of frequency response of electrical conductivity and dielectric permittivity in the carbon nanotube/polymer composites. Carbon 54:105-112. doi:10.1016/ j.carbon.2012.10.068

Shehzad K et al (2013b) All-organic PANI-DBSA/PVDF dielectric composites with unique electrical properties. J Mater Sci 48:3737-3744. doi:10.1007/s10853-013-7172-5

Shehzad K, Zha J-W, Zhang Z-F, Yuan J-K, Dang Z-M (2013c) Piezoresistive Behavior of Electrically Conductive Carbon Fillers/Thermoplastic Elastomer Nanocomposites. J Adv Physics 2:70-74

Shehzad K et al (2014) Influence of carbon nanotube dimensions on the percolation characteristics of carbon nanotube/polymer composites. J Appl Phys 116:064908

Stoyanov H, Brochu P, Niu X, Lai C, Yun S, Pei Q (2013) Long lifetime, fault-tolerant freestanding actuators based on a silicone dielectric elastomer and self-clearing carbon nanotube compliant electrodes. RSC Advances 3:2272-2278. doi:10.1039/ C2RA22380E

Wang L, Dang ZM (2005) Carbon nanotube composites with high dielectric constant at low percolation threshold. Appl Phys Lett 87:042903

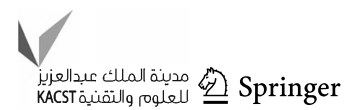


Wang D, Zhou T, Zha J-W, Zhao J, Shi C-Y, Dang Z-M (2013) Functionalized graphene-BaTiO3/ferroelectric polymer nanodielectric composites with high permittivity, low dielectric loss, and low percolation threshold. J Mater Chem A 1:6162-6168

Yadav SK, Mahapatra SS, Cho JW (2013) Tailored dielectric and mechanical properties of noncovalently functionalized carbon nanotube/poly [styrene-b-(ethylene-co-butylene)-b-styrene] nanocomposites J Appl Polym Sci

Yang C, Lin Y, Nan CW (2009) Modified carbon nanotube composites with high dielectric constant, low dielectric loss and large energy density. Carbon 47:1096-1101. doi:10.1016/j. carbon.2008.12.037

Yu Z, Yuan W, Brochu P, Chen B, Liu Z, Pei Q (2009) Large-strain, rigid-to-rigid deformation of bistable electroactive polymers. Appl Phys Lett. doi:10.1063/1.3263729

Zeng Y, Liu P, Du J, Zhao L, Ajayan PM, Cheng H-M (2010) Increasing the electrical conductivity of carbon nanotube/polymer composites by using weak nanotube-polymer interactions. Carbon 48:3551-3558. doi:10.1016/j.carbon.2010.05.053 